\title{
Role of charge separation mechanism and local disorder at hybrid solar cell interfaces
}

\author{
Philipp Ehrenreich, ${ }^{1}$ Thomas Pfadler, ${ }^{1}$ Francis Paquin, ${ }^{2}$ Laura-Isabelle Dion-Bertrand, ${ }^{2}$ Olivier Paré-Labrosse, ${ }^{2}$ Carlos Silva, ${ }^{2}$ \\ Jonas Weickert, ${ }^{1, *}$ and Lukas Schmidt-Mende ${ }^{1}$ \\ ${ }^{1}$ Department of Physics, University of Konstanz, POB 680, 78467 Constance, Germany \\ ${ }^{2}$ Département de physique et Regroupement québécois sur les matériaux de pointe, Université de Montréal, \\ C.P. 6128, Succursale centre-ville, Montréal, Québec, Canada
}

(Received 11 October 2014; published 12 January 2015)

\begin{abstract}
Dye-sensitized metal oxide polymer hybrid solar cells deliver a promising basis in organic solar cell development due to many conceptual advantages. Since the power conversion efficiency is still in a noncompetitive state, it has to be understood how the photocurrent contribution can be maximized (i.e., which dye-polymer properties are most beneficial for efficient charge generation in hybrid solar cells). By the comparison of three model systems for hybrid solar cells with $\mathrm{TiO}_{2}$-dye-polymer interfaces, this paper was aimed at elucidating the role of the exact mechanism of charge generation. In the exciton dissociation (ED) case, an exciton that is generated in the polymer is split at the dye-polymer interface. Alternatively, this exciton can be transferred to the dye via an energy transfer (ET), upon which charge separation occurs between dye and $\mathrm{TiO}_{2}$. For comparison, the third case is included in which the high lowest unoccupied molecular orbital of the dye does not allow exciton separation or ET from the dye to the polymer, so that the dye only is responsible for charge generation. To separate effects owing to differences in energy levels of the involved materials from the impact of local order and disorder in the polymer close to the interface, this paper further comprises a detailed analysis of the polymer crystallinity based on the $\mathrm{H}$-aggregate model. While the massive impact of the poly(3-hexylthiophene) crystallinity on device function has been outlined for bare metal oxide-polymer interfaces, it has not been considered for hybrid solar cells with dye-sensitized $\mathrm{TiO}_{2}$. The results presented here indicate that all dye molecules in general influence the polymer morphology, which has to be taken into account for future optimization of hybrid solar cells. Apart from that, it can be suggested that ED on the polymer needs an additional driving force to work efficiently; thus, energy transfer seems to be currently the most promising strategy to increase the polymer photocurrent contribution.
\end{abstract}

DOI: 10.1103/PhysRevB.91.035304

PACS number(s): 73.20.At

\section{INTRODUCTION}

Hybrid metal oxide-polymer cells try to combine advantages of fully organic solar cell concepts with beneficial properties of inorganic compounds that are used as electron acceptors. Materials like $\mathrm{TiO}_{2}$ exhibit a large bandgap, filtering the ultraviolet (UV) light portion of the solar spectrum which is most often harmful for organic materials in ambient conditions [1,2]. The chemical stability in combination with controllable phase separation of a low-cost material adheres to the concept of a long-term stable, nontoxic solar cell application with well-controlled properties [3,4]. Within this solar cell concept the nanostructured $\mathrm{TiO}_{2}$ electrode is rendered highly absorbing by sensitization with a self-assembled monolayer of dye molecules. As organic hole transporting material (HTM) an absorbing conjugated polymer is used rather than a transparent HTM-like spiro-OMeTAD $\left[2,2^{\prime}, 7,7^{\prime}\right.$-tetrakis-( $N, N$-di- $p$-methoxyphenylamine $) 9,9^{\prime}$-spirobifluorene] to enable additional photocurrent contribution and enhance the overall device absorptivity [5]. Ideally, the polymer absorbs complementary to the dye, and both exhibit high extinction coefficients so that a broad range of the incident solar flux can be harvested.

When a hybrid solar cell is operated, excitons generated in the dye molecule are efficiently separated via electron injection into the metal oxide, while holes are transferred to the HTM [Fig. 1(c)]. Despite the apparent advantage of additional

*Corresponding author: Jonas.Weickert@uni-konstanz.de photocurrent contribution of the polymer compared to liquid electrolyte dye-sensitized solar cells (DSSCs), efficiencies are currently relatively low for hybrid solar cells. One reason for this is seen as an ineffective pore filling of the mesoporous metal oxide with the solid state HTM, especially for films thicker than few microns [6,7]. Consequently, liquid electrolyte DSSCs are a factor of 5-10 thicker than hybrid solar cells, and dye photocurrent contributions are much higher. Most importantly, the role of the polymer photocurrent contribution has been widely neglected. This is reflected in often marginal polymer contributions to the external quantum efficiency (EQE) spectra and, in some instances, where the polymer is implemented as a completely noncontributing hole transporter only. Here the device efficiency is further lowered by parasitic absorption due to overlaps in dye and polymer absorption spectra [5,8]. Accordingly it is important to enhance the polymer photocurrent contribution as much as possible to achieve higher efficiencies, to fully make use of the potential of hybrid solar cells. For this purpose, it is imperative to identify material properties that are responsible for fundamental limitations in photocurrent generation.

When analyzing a certain hybrid metal oxide-dye-polymer material combination, it is necessary to determine which charge generation process is most efficient in terms of charge separation, especially at the dye-polymer interface. As presented in Fig. 1(c), there are two possibilities for how a photoexciton originating from the polymer can be separated. First, excitons can diffuse through the polymer to the dye interface where they dissociate if the energy level offset is suitable for cracking the binding energy of the exciton. Second, 

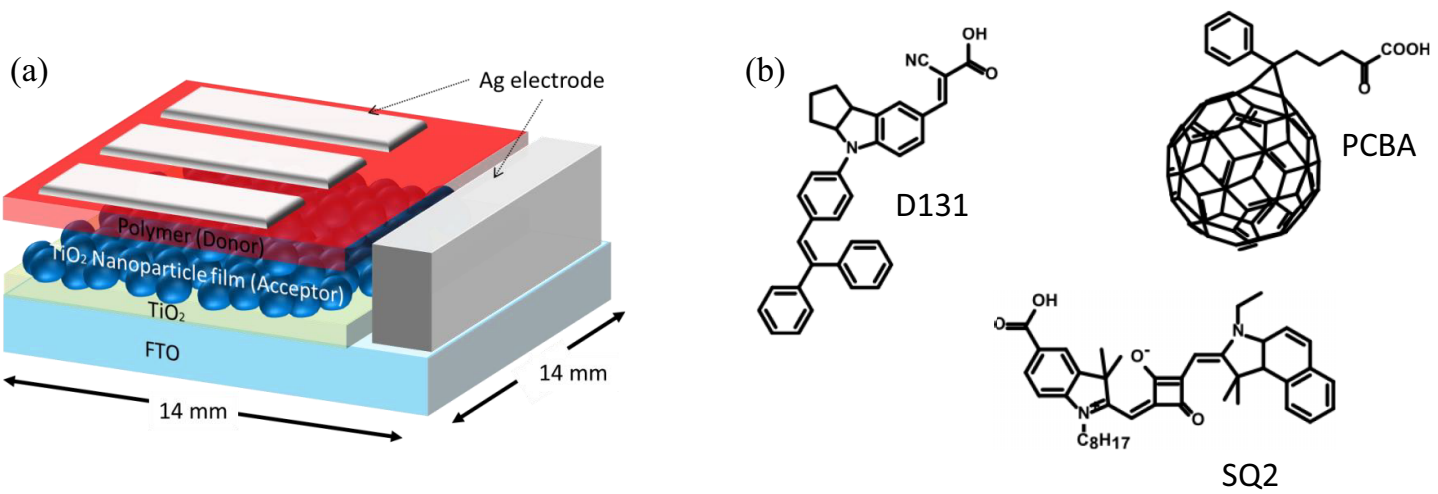

(c)

Dye photocurrent contribution

Polymer photocurrent contribution
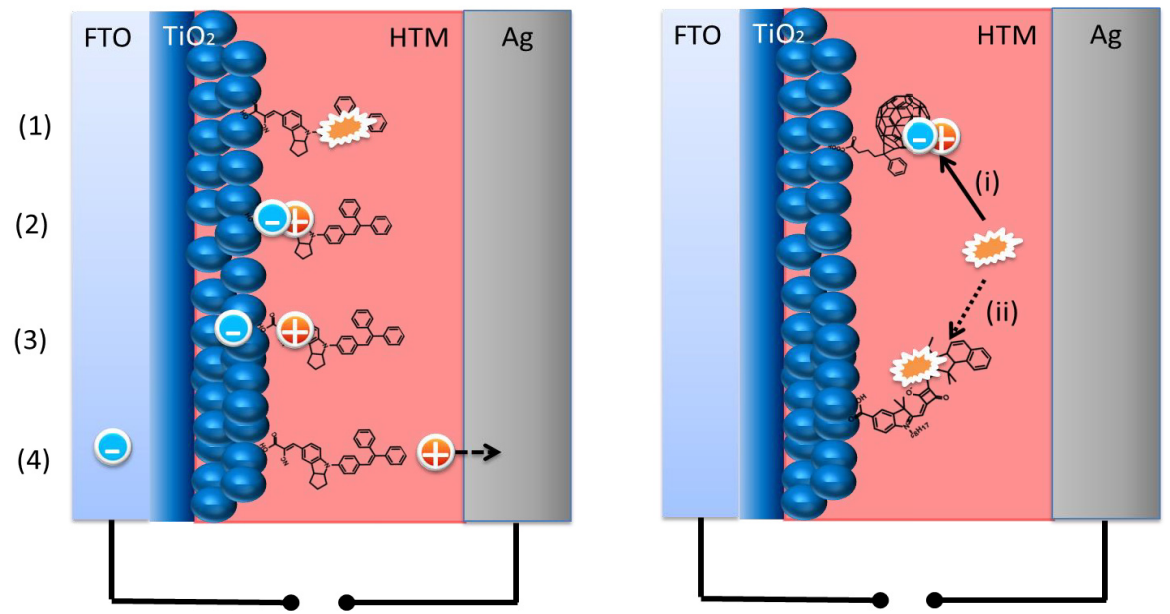

FIG. 1. (Color online) (a) Schematic of hybrid solar cell architecture. (b) Dye molecules that are under investigation as model systems. (c) Charge generation mechanism in a dye (left): (1) exciton generation after photoexcitation, (2) exciton diffusion to the electron-accepting $\mathrm{TiO}_{2}$ interface, (3) electron injection into $\mathrm{TiO}_{2}$, and (4) hole injection into the $\mathrm{HTM}$ and charge extraction; charge generation mechanism in the polymer (right). After photoexcitation, an exciton can either be dissociated at a dye/metal oxide interface (i) or it can be transferred via FRET to the dye molecule, from which it follows the mechanism on the left.

the exciton energy can be transferred via Förster resonant energy transfer (FRET) from the polymer to the dye $[9,10]$. As a matter of principle, it is still unclear to which extent solar cell properties are affected by these different mechanisms. A systematic investigation of the fundamental implication for device operation of these mechanisms is crucial to understand major advantages, drawbacks, and loss mechanisms, particularly for future material design and composition. This paper addresses this question by comparing three model systems for (1) dye only (DO) photocurrent contribution, (2) exciton dissociation (ED) in the polymer at the dye interface, and (3) FRET of the exciton from the polymer to the dye. A HTM poly(3hexylthiophene) (P3HT) is used due to its widely known and extensively studied properties [11-15]. Furthermore, it is the most widely employed polymeric HTM in hybrid solar cells and thus is well suited to serve as a model HTM. As for many conjugated polymers, the degree of crystallinity in the polymer phase massively influences the electronic properties of P3HT, so that its implementation in our model system enables us to investigate structural influences on the electronic properties of our devices. Conclusions on structural as well as energetic differences can be obtained [11,12] to avoid error-prone interpretations when not taking this very important factor for organic semiconductors into account. To do so, this paper also discusses the influence of the dye molecule on the arrangement and intermolecular communication of the polymer. The polymer weight is chosen to be intermediate, at $56 \mathrm{~kg} \mathrm{~mol}^{-1}$, to deliver high intermolecular interaction but also sufficiently interconnected crystalline polymer regions, which is a necessary condition for efficient transport [16].

In combination with $\mathrm{P} 3 \mathrm{HT}$, we employ the following dye molecules as model systems for DO, ED, and energy transfer (ET): The indoline dye D131 is used as the suitable model dye for a DO system [5,7] since it exhibits a high extinction coefficient of $48000 \mathrm{M}^{-1} \mathrm{~cm}^{-1}$ and does not enable any polymer photocurrent contribution in combination with $\mathrm{P} 3 \mathrm{HT}$ [17].

[6,6]-Phenyl C61 butyric acid (PCBA) is chosen for ED since it functions as a dye molecule for which ED at the dyepolymer interface is possible. Furthermore, as a fullerene-type molecule, good electron-accepting properties can be expected that favor electron injection from the polymer into the dye 
molecule. As has been previously shown, PCBA enhances the polymer contribution compared to interfaces not sensitized to either $\mathrm{ZnO}$ or $\mathrm{TiO}_{2}$ in combination with P3HT [18,19].

For the ET system, the squaraine dye SQ2 is chosen. Similar squaraine dyes have been successfully implemented in highefficiency hybrid solar cells, and direct evidence for a rapid FRET from P3HT to the dye has been provided through a transient absorption study by Huang et al. [20,21].

The chemical structure of all dyes is summarized in Fig. 1(b), and in the following, the different systems shall be referenced by their appropriate acronyms.

Using a combination of optical and electronic characterization techniques, including light intensity-dependent current density-voltage $J(V)$ measurements and quasisteady state photoinduced absorption [continous wave (cw)-PIA] experiments, we draw a picture of the interplay between local polymer structure and exciton separation at hybrid interfaces.

This paper is organized as follows: In Sec. II. sample preparation details and measurement techniques are explained. Section III deals with solar cell characteristics obtained in $J(V)$ and EQE measurements. These results are then further investigated and illuminated with respect to the polymer morphology under the scope of the $\mathrm{H}$-aggregate model. This part contains a short introduction and summary into fundamental and basic ideas of that theoretical description. This comparison is especially possible for this model system investigation because we keep basic processing conditions constant while changing the dye molecules only such that differences in the polymer morphology can be directly related to the influences which are introduced by this surface modification. In the last part of this section we present results of PIA measurements that solidify the assumption of a more efficient energy transfer compared to charge separation. Section IV contains a discussion of the results, and the conclusion is found in Sec. V.

\section{EXPERIMENTAL METHODS}

\section{A. Sample preparation}

Fluorine-doped $\mathrm{SnO}_{2}$ (FTO)-coated glass substrates (Solaronix, $15 \Omega \square^{-1}$ ) were cleaned successively in an ultrasonic bath of soap water, acetone, and 2-propanol for $5 \mathrm{~min}$ and dried in a nitrogen stream. Via spray pyrolysis at $450{ }^{\circ} \mathrm{C}$ of a diisopropoxytitanium bis(acetylacetonate) solution (SigmaAldrich) in ethanol (1:10), an approximately 75-nm-thick $\mathrm{TiO}_{2}$ compact layer was deposited and annealed for another 15 min at $450{ }^{\circ} \mathrm{C}$. On top, a $\mathrm{TiO}_{2}$ nanoparticle (Dysole $18 \mathrm{NR}-\mathrm{T}$ ) film was spin coated from an ethanol solution at $1500 \mathrm{rpm}$ for $60 \mathrm{~s}$. The films were then sintered at $450{ }^{\circ} \mathrm{C}$ for 60 min and subsequently placed in an aqueous $\mathrm{TiCl}_{4}$ solution $(20 \mathrm{mM})$ at $70^{\circ} \mathrm{C}$ for $1 \mathrm{~h}$. After a second sintering step at $450{ }^{\circ} \mathrm{C}$ for $60 \mathrm{~min}$, the samples were submerged into the appropriate dye bath. To saturate all free surface vacancies at the metal oxide surface and realize its beneficial energetic effects on the conduction band on $\mathrm{TiO}_{2}$ due to its dipole moment [22,23], 4-tert-butylpyridine (tBP, Sigma-Aldrich) was spin coated at $2000 \mathrm{rpm}$ for $60 \mathrm{~s}$. In combination with D131, this sulphonyl solution is mixed 1:1 with lithium bis(trifluoromethane sulphone)imide (LiTFSI). It has been shown that LiTFSI increases the pore-filling fraction and the charge conductivity for D131-based cells [7], which is especially needed for a reasonable fill factor and photocurrent due to the optimized charge transfer nature of the excited state [24]. The concentration for both additive solutions was $50 \mathrm{mM}$ in acetonitrile. Finally, P3HT (Rieke, $\mathrm{M}_{\mathrm{W}}=56 \mathrm{~kg} \mathrm{~mol}^{-1}$, regioregularity $>96 \%$ ) was spin coated from a dichlorobenzene solution at a concentration of $20 \mathrm{mg} \mathrm{ml}^{-1}$ at $1500 \mathrm{rpm}$ for $120 \mathrm{~s}$. For the full solar cell architecture, an 80-nm-thick Ag layer was thermally evaporated as the electrode, with a pixel size of $0.125 \mathrm{~cm}^{2}$. In addition for absorption and photoluminescence (PL) measurements, samples were fabricated on microscope glass slides instead of FTO substrates.

\section{B. Measurement techniques}

EQE and $J(V)$ measurements were performed by using a Keithley 2400 source meter. For $J(V)$ measurements the samples were illuminated with a Lot Oriel AM1.5G solar simulator, while a sweep rate of $0.02 \mathrm{~V} \mathrm{~s}^{-1}$ has been applied from reverse to forward bias. Cells were kept in a light-tight were sample holder, covering back and sides of the sample, and illuminated through a shadow mask with an active cell area of $0.125 \mathrm{~cm}^{2}$. A KG5 filter-equipped Fraunhofer Institute for Solar Energy Systems (ISE) certified Si reference cell was used for calibration. If required, light intensity was reduced using appropriate neutral-density filters. For EQE measurements, a Lot Oriel Omni 150 monochromator was used to scan the spectral current output of the solar cell.

Absorption spectra were recorded with an Agilent Technologies 8453 UV-VIS spectrometer. For PL experiments, samples were excited with a continuous wave laser $(50 \mathrm{~mW}, 532 \mathrm{~nm})$ that is mechanically chopped at a frequency of $170 \mathrm{~Hz}$. For detection, the probe spectrum was scanned with a Princeton Instruments (PI) SP2300 monochromator, while a Si/PbS-025/020-TE2-H photoreceiver was connected to an SR830 lock-in amplifier.

The exact description of the cw-PIA setup is described elsewhere [25]. Briefly, it is a background-free dual optical modulation scheme, in which the pump laser is modulated with a frequency of $170 \mathrm{~Hz}$ and the probe beam at $139 \mathrm{~Hz}$. As probe source, a 250-W tungsten-halogen lamp (Oriel) is coupled to a $300-\mathrm{mm}$ focal length monochromator (PI SP2300). The same spectrometer was used in the detection line in front of a photoreceiver consisting of a Peltier-cooled, sandwiched $\mathrm{Si} / \mathrm{PbS}$ diode (Electro-Optical Systems S/PBS025/020-TE2-H). The change in transmission was recorded at the sum frequency using two digital lock-in amplifiers (Stanford Research Systems SR830 and SR810) to avoid any parasite signal coming from the pump contribution only, such as PL.

\section{RESULTS AND ANALYSIS}

\section{A. Current-density voltage $J(V)$ characteristics}

In Fig. 2, typical $J(V)$ curves are presented which are representative for general observations within these model systems. Statistical values can be found in the Supplemental 


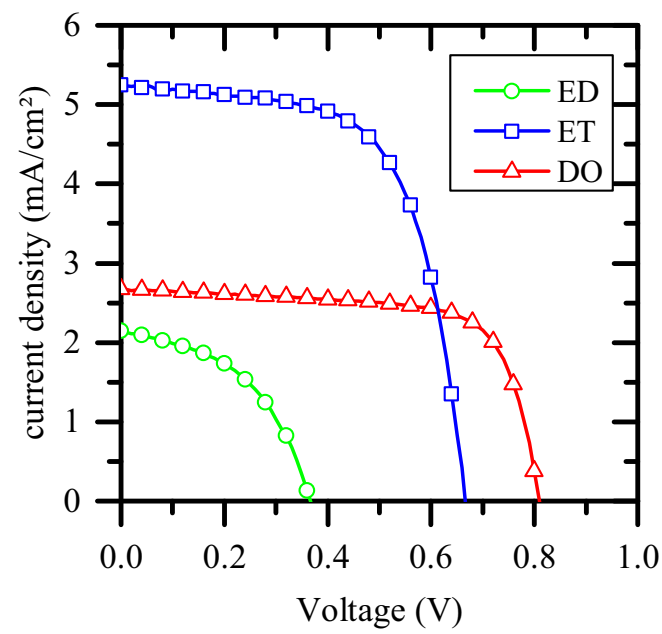

FIG. 2. (Color online) Characteristic $J(V)$ curves for the ED, (green circles), ET, (blue squares) and DO, (red triangles) model systems with P3HT as HTM.

Material [26] to show its reproducibility [27]. Devices are not optimized for high efficiencies in this work since general claims and conclusions would become difficult if critical device parameters like the polymer capping layer thickness are pushed to the limit.

Morphology considerations especially could be very prone to error if different treatments on the individual concepts would be applied. It has been shown that the current especially depends a lot on the titanium thickness $[7,8]$ and annealing treatments $[18,19,28]$. Additionally, the amount of dye absorption can be considerably different. Note, however, that fill factors $(\mathrm{FFs})$ and open circuit voltages $\left(V_{\mathrm{OC}}\right)$ are comparable to values reported in the literature [28]. We therefore conclude that the fundamental mechanisms of exciton diffusion, exciton separation, and charge carrier transport and recombination can be considered to be identical to those in devices with optimized efficiencies (i.e., optimized photocurrents). Note that invariant fabrication steps are essential for comparisons among the systems, such that differences in the charge generation can be explained by exchange of the dye monolayer only.

Corresponding solar cell results are summarized in Table I. When comparing the different model systems with each other, it is obvious that the ET system shows the highest efficiencies, mainly owing to the much higher short-circuit current density $\left(J_{\mathrm{SC}}\right)$. For SQ2-based cells, the photocurrent is twice as high as for the other two systems, while $\mathrm{FF}$ and $V_{\mathrm{OC}}$ are significantly higher in the DO system. The observed FF of $75 \%$ and $V_{\mathrm{OC}}$ of $0.83 \mathrm{~V}$ are an indication that charge extraction

TABLE I. Solar cell characteristics exemplary for the three systems under investigation.

\begin{tabular}{lccc}
\hline \hline & PCBA (ED) & SQ2 (ET) & D131 (DO) \\
\hline$n(\%)$ & 0.37 & 2.22 & 1.53 \\
$\mathrm{FF}(\%)$ & 47 & 63 & 75 \\
$V_{\mathrm{OC}}(V)$ & 0.37 & 0.67 & 0.83 \\
$J_{\mathrm{SC}}\left(\mathrm{mA} / \mathrm{cm}^{2}\right)$ & $\mathbf{2 . 1 4}$ & $\mathbf{5 . 3}$ & $\mathbf{2 . 7}$ \\
\hline \hline
\end{tabular}

works efficiently and polaron recombination is not limiting the device performance. In contrast, both values are generally low for the ED system. The FF can be negatively influenced by many factors, most prominently imbalanced mobilities, bad morphology between the active layer and the electrodes, the presence of electrical dipoles, and larger potential barriers [29]. To focus on the differences in charge extraction efficiencies among the three systems under investigation, we have used results from light intensity-dependent $J(V)$ measurements to calculate the charge collection probability $P_{\mathrm{c}}(I, V)$ introduced by Cowan et al. [30] and Street et al. [31]. $P_{\mathrm{c}}(I, V)$ is given by the light intensity $(I)$-and voltage $(V)$-dependent photocurrent corrected by the dark current and normalized to the saturation current (i.e., the photocurrent that is independent on the applied voltage $V_{\mathrm{S}}$ ):

$$
P_{\mathrm{c}}(I, V)=\left|\frac{J_{\mathrm{ph}}(I, V)-J_{\mathrm{dark}}(V)}{J_{\mathrm{ph}}\left(I, V_{\mathrm{s}}\right)}\right|
$$

The charge collection probability as a function of applied voltage is shown in Fig. 3 for the three systems. In an ideal case the light intensity dependent $J(V)$ curves would follow the same behavior from $V_{\mathrm{S}}=-0.3 \mathrm{~V}$ close to the maximum power point. This behavior can be observed if the photocurrent is linearly dependent on the charge generation rate [31,32]. In fact, this is the case for the DO system,

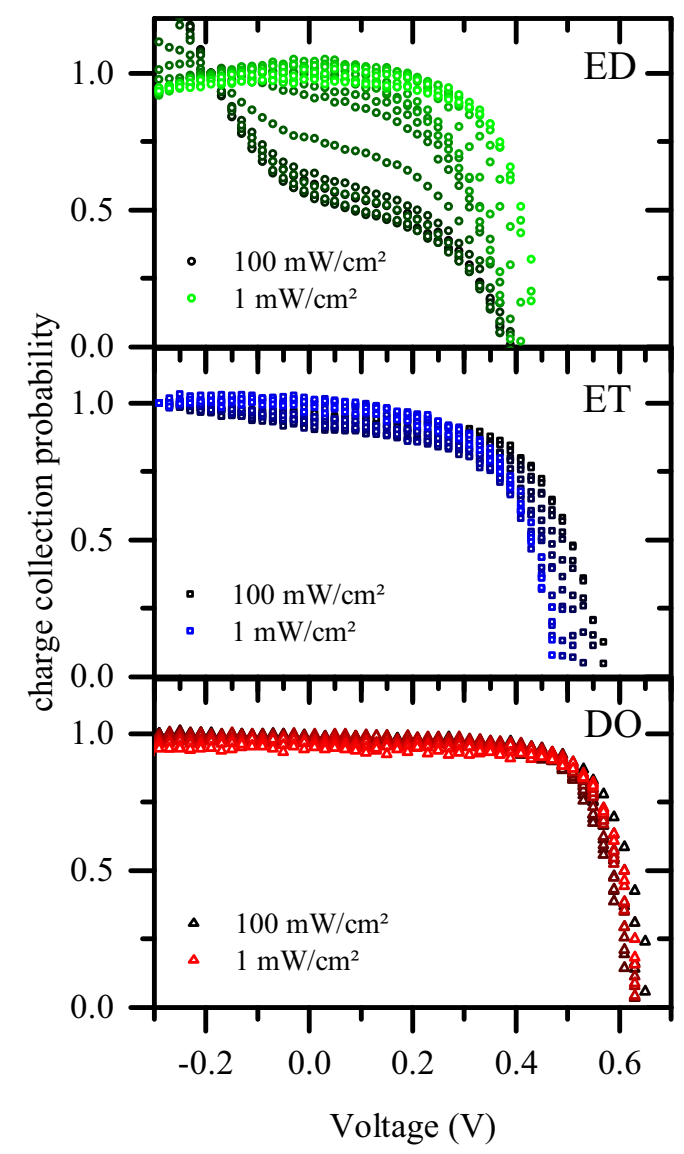

FIG. 3. (Color online) Charge collection probability calculated with formula (1) for light intensity dependent $J(V)$ measurements ranging from 1 to $100 \mathrm{~mW} / \mathrm{cm}^{2}$. Top: ED; middle: ET; bottom: DO. 
implying the dominant role of first-order (monomolecular) recombination. For ET the situation is different. Already from $V_{\mathrm{S}}$ the charge collection probability is decreasing, indicating an electric field-assisted charge separation in the device. The charge density rate is overlapped by a higher order process that is enhanced in forward bias, resulting in a split in the $J(I, V)$ curves. This observation is even more dramatic for the ED system. Especially for higher light intensities, significant deviations from the ideal case are apparent. The charge collection probability gets S-shaped, which possibly is an indication of charge accumulation in the device, most likely at the donor-acceptor interface. Due to this inefficient charge separation, bimolecular recombination is enhanced. However, it is not possible to evaluate whether trap states or poor charge separation due to insufficient internal electric field are responsible for this behavior.

\section{B. External quantum efficiency}

The reason for the relatively high efficiency obtained for the ET system can be found in a two times higher $J_{\mathrm{SC}}$ compared to the other two systems. To explain this result, we compared the EQE spectra of the three systems (filled areas in Fig. 4). Absorption spectra of the pristine polymer (dotted line), pristine dye (dashed-dotted line), and full structure (solid line) are plotted as references to resolve the photocurrent contribution of the individual components of the solar cell structure.

For the ET system, P3HT has the highest contribution to the photocurrent. Current generation from the polymer can be seen even below $450 \mathrm{~nm}$, which corresponds to weakly aggregated polymer domains [12,33,34]. This is not surprising since it could be shown that ED in the polymer is driven or even enhanced by extrinsic energetic effects at the interface between highly crystalline aggregates and nonaggregated domains [35]. For the DO system, the polymer does not contribute to the photocurrent, as expected from the alignment of the lowest unoccupied molecular orbitals (LUMOs) of D131 and P3HT $[5,7]$. This energy level alignment hinders dissociation of excitons in the P3HT at the dye-polymer interface. Although the maximum EQE reaches $55 \%$ at $420 \mathrm{~nm}$ in the DO system, which is higher than the peak EQE of the ET system, the latter profits from much larger polymer contributions. Interestingly, this polymer photocurrent contribution is weaker for the ED system. Due to an absorption overlap of the polymer with the dye, we were not able to resolve whether the nonaggregated domains were also more efficient in terms of ED for a dye-sensitized metal oxide surface. Transient absorption and time-resolved PL experiments are expected to help resolve this issue, but they are not in the scope of the present paper.

\section{UV/Visible absorption and PL-morphology investigation}

The polymer structure in an aggregated film is very complex and not trivial to resolve due to its nanometer scale. Different packing and chain-chain interactions can significantly influence the electronic properties of P3HT [36-38]. Important parameters like the charge carrier mobility, charge density, and exciton transport are affected by the relevance of intermolecular interaction compared to intramolecular polymer forces

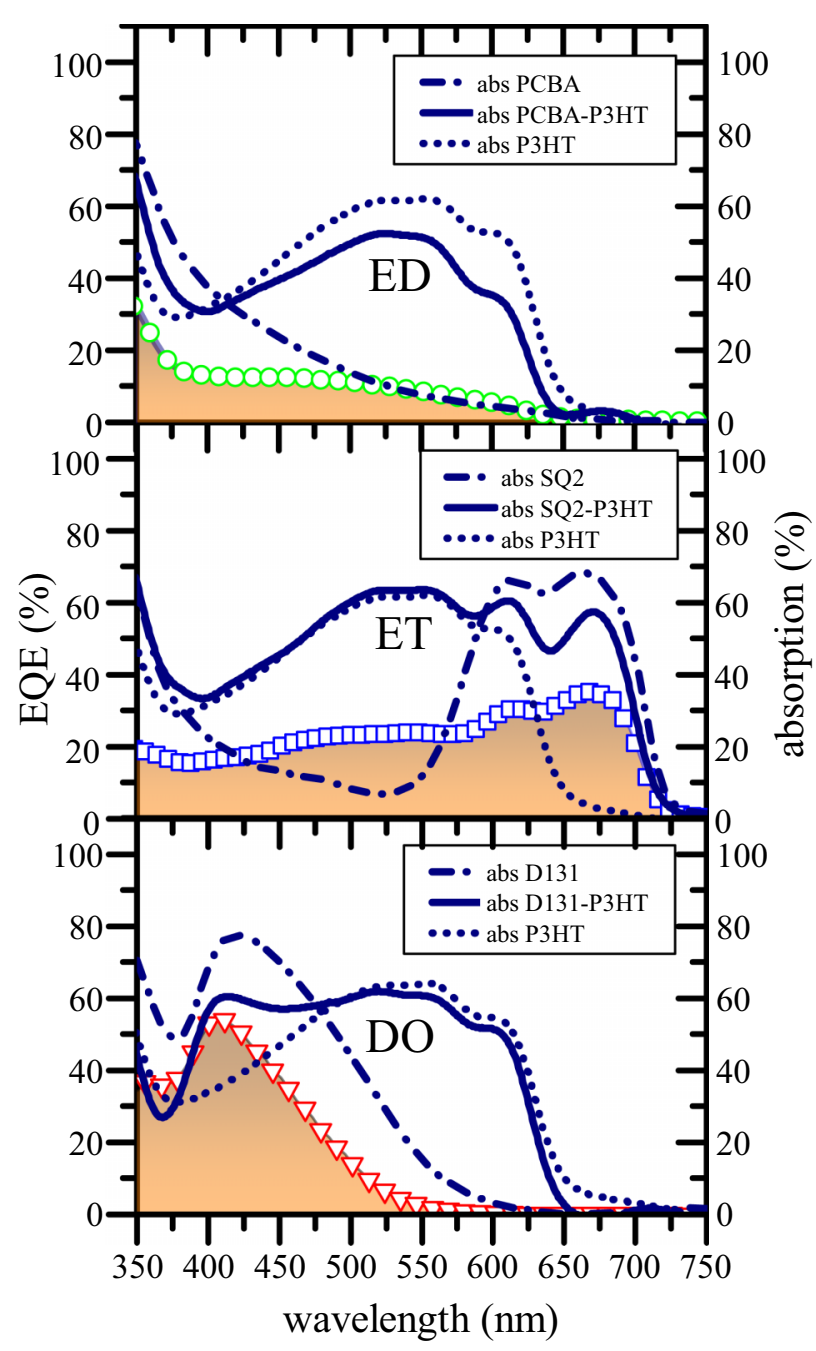

FIG. 4. (Color online) EQE spectra of the three model systems. Top: ED (green circles), middle: ET (blues squares), bottom: DO (red triangles); in all graphs the absorption spectra of the compound, as well as the pristine materials, are included to demonstrate the origin of the photocurrent.

[11,12,37,39-41]. By self-organization regioregular P3HT (rrP3HT) forms a two-dimensional (2D)-lamellae structure that is perpendicular to a substrate's surface with strong interchain interaction. This self-organization process depends on many factors, like solvent concentration, solvent boiling point, temperature, environmental conditions, molecular weight, and so on $[11,14,41]$. Most importantly, the polymer order can be directly influenced by the properties of the coated surface. For this reason it is essential to know whether and to what extent dye sensitization influences the polymer morphology. To our knowledge, there is only little research on the dye-induced polymer morphology at hybrid interfaces, although this issue can strongly influence the electronic properties at the interface. Since we hold the processing conditions constant over all model systems, we are able to resolve this fundamental question. It has been shown that the surface modification of $\mathrm{TiO}_{2}$ with a dye molecule has a large impact on solar cell performance and ED due to the introduction of a surface dipole, which shifts the conduction band $[18,22,42]$. However, similar 
effects on charge separation, recombination, and transport processes have to be taken into account for the other part of the dye-sensitized hybrid interface-namely, the polymer. In the following, we address this question by analyzing UV/Visible (UV/Vis) and PL spectra using an H-aggregate model for P3HT [11,12,14,37,39,43-45]. Within this model the morphology is probed by investigating the intermolecular interaction, or rather coupling between the dipole moments of polymer chains in close proximity. Negative coupling is present between neighboring chromophores if they are in a head-to-tail configuration, whereas a side-by-side orientation results in a positive coupling [38]. As a consequence of this resonant Coulombic interaction, vibronic energy levels split with respect to certain selection rules for optical transitions. This in turn leads to absorption/emission characteristics that differ significantly from the simple Franck-Condon progression that usually describes the optical transitions in organic semiconductors when only taking exciton-phonon coupling into account. A measure for the intermolecular interaction energy $J_{\text {inter }}$ is the exciton bandwidth $W=4 J_{\text {inter }}$. As has been introduced by Clark et al. [11,12], $W$ can be determined by the ratio of the $A_{0-0}$ to $A_{0-1}$ peak in an $\mathrm{H}$-aggregate when assuming a Huang-Rhys factor $\lambda$ of unity:

$$
\frac{A_{0-0}}{A_{0-1}} \approx\left(\frac{1-0.24 W / \hbar \omega_{0}}{1-0.073 W / \hbar \omega_{0}}\right)^{2}
$$

The assumption of $\lambda=1$ is reasonable for the molecular weight $56 \mathrm{~kg} \mathrm{~mol}^{-1}$, considering the study of Paquin et al. [14]. $\hbar \omega_{0}=0.175 \mathrm{meV}$ in this formula describes the energy of the $\mathrm{C}=\mathrm{C}$ intramolecular vibrational stretching mode to which the electronic transition couples [46].

As can be seen in Fig. 5 and summarized in Table II, the polymer morphology deposited on a flat glass substrate or a mesoporous $\mathrm{TiO}_{2}$ matrix is either not or only marginally different. Both spectra coincide, and the 0-0/0-1 vibronic transitions are positioned at the same energies of 2.05 and $2.22 \mathrm{eV}$, respectively. The relatively slow drop-off on the high-energy shoulder can be explained with contributions from $\mathrm{TiO}_{2}$ and electronically uncoupled chromophores, or rather domains that are of a non-H-aggregate type $[12,16]$, that cannot be avoided in a real polymer film. The similarity of both spectra implies that the inorganic metal oxide does not have a decisive influence on the bulk polymer morphology. Note that this observation does not contradict findings by Kandada et al. [15], who report a noticeable amount of disorder introduced by the mesoporous surface, because there is a large dependence

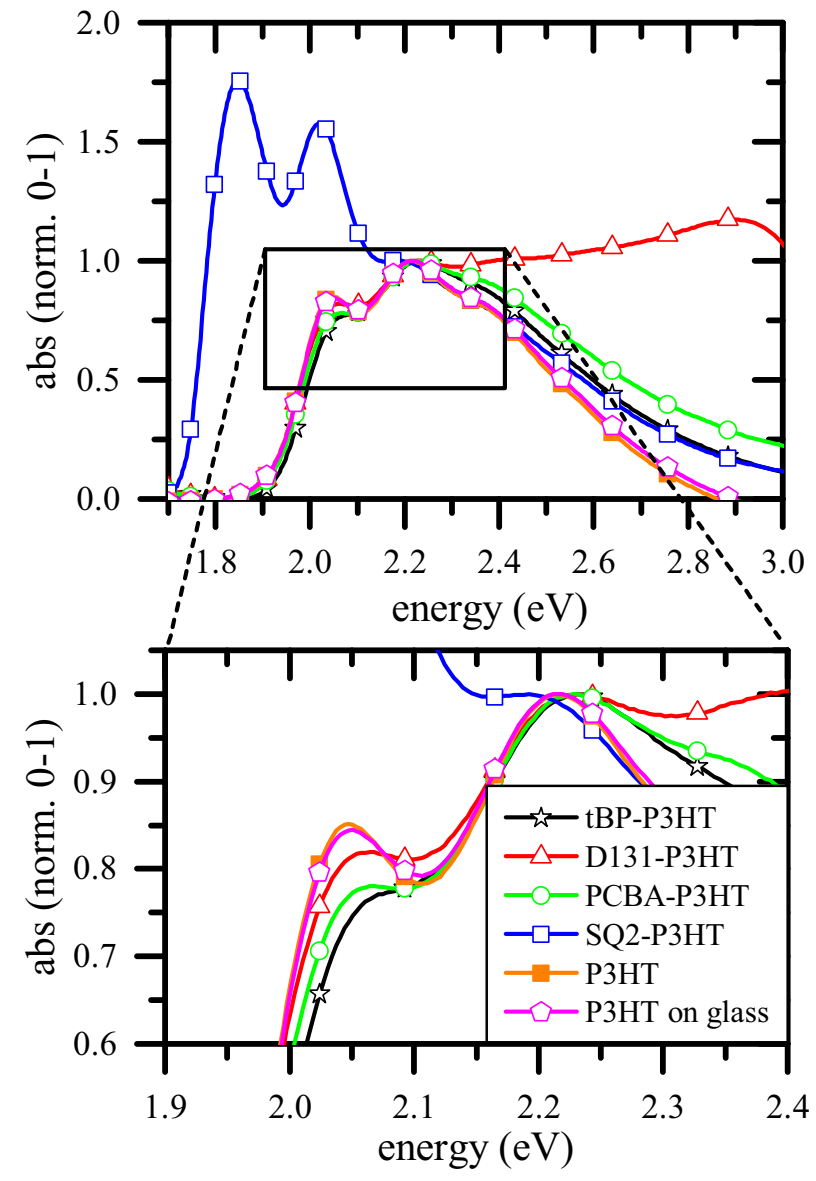

FIG. 5. (Color online) UV/Vis absorption spectra of P3HT on different dye-sensitized $\mathrm{TiO}_{2}$ substrates normalized to the 0-1 transition. Top: full spectrum; bottom: zoom-in (indicated in black square) to resolve differences in the 0-0 transition, relevant for the determination of the exciton bandwidth using Eq. (2).

on the amount of pore filling, different molecular weights, process conditions, and $\mathrm{TiO}_{2}$ composition.

However, the more important result of our paper is that a tBP-modified surface leads to an increase in the exciton bandwidth $W$ by over $35 \%$. Besides, the spectrum is not only blueshifted by $20 \mathrm{meV}$ but also the nonaggregate signature is enhanced. The PCBA does not change very much, except that it seems to reduce the spectral blueshift again. The same behavior can be observed for D131, for which $\mathrm{W}$ is also reduced. However, in this case a spectral overlap between

TABLE II. Exciton bandwidth $W$ for the samples under investigation derived by Gaussian fits of room temperature absorption spectra shown in Fig. 5. The energetic positions of the $0-0$ and $0-1$ vibronic transitions are included to show a spectral blueshift when modifying the $\mathrm{TiO}_{2}$ surface.

\begin{tabular}{lccc}
\hline \hline Sample & $W(\mathrm{meV})$ & $0-0$ transition $(\mathrm{eV})$ & $0-1$ transition $(\mathrm{eV})$ \\
\hline P3HT (on glass) & $44.7 \pm 1.5$ & 2.05 & 2.22 \\
P3HT & $42.9 \pm 1.5$ & 2.05 & 2.22 \\
tBP-P3HT & $67.4 \pm 3.2$ & 2.07 & 2.23 \\
PCBA-tBP-P3HT & $64.9 \pm 1.6$ & 2.06 & 2.23 \\
D131/LiTFSI -tBP -P3HT & $52.2 \pm 1.6$ & 2.06 & 2.23 \\
SQ2-tBP-P3HT & - & - & 2.19 \\
\hline
\end{tabular}


the dye and the polymer absorption has to be considered. For this reason the determination of $W$ is prone to error, and an overestimation of the $0-1$ transition can be expected. For PCBA this effect is almost negligible, since the higher spectral weight at $2.23 \mathrm{eV}$ compared to $2.06 \mathrm{eV}$ only leads to an overestimation of roughly $1 \%$, which is below the standard deviation of the determination of $W$. For D131 the effect is more relevant, with an overestimation of 9-10\% of the same transition. The effect on $W$ would therefore be enhanced even, and the values in Table II can be seen as an upper limit. A qualitative analysis of the high-energy shoulder cannot be made for the same reason, although the spectral redshift of only $10 \mathrm{meV}$ indicates that dye molecules have improved the crystallinity of P3HT near the interface when compared to the pure tBP case. Unfortunately this analysis cannot be made for the ET system since the 0-0 transition of P3HT overlaps with the dye absorption. This overlap exists also to some extend in the high-energy part of the spectrum. The 0-1 transition, which is not affected, seems to be redshifted compared to neat P3HT films, which indicates a higher degree of aggregation consistent with the observations for the other dyes.

Further information on the structural conditions can be gained by low-temperature PL measurements. Within the $\mathrm{H}$-aggregate model, energetic, or rather conformational, disorder within $\pi$-stacked crystalline domains can limit the intermolecular exciton coherence and make 0-0 transitions possible. At $T=10 \mathrm{~K}$ it is therefore possible to investigate the relevance of disorder in such films, because vibrations are frozen and temperature effects are almost eliminated. In other words, the spectral weight of the $0-0$ transition is a measure for intermolecular interaction and, on the other hand, defects in the crystal order. As has been shown earlier [12,14,44], the Franck-Condon progression can still be applied, although it has to be modified concerning the disorder issue:

$$
\begin{aligned}
I(\omega) \propto & (\hbar \omega)^{3} n^{3}(\omega) e^{-\lambda_{\text {eff }}^{2}}\left[\alpha \Gamma\left(\hbar \omega-E_{0}\right)\right. \\
& \left.+\sum_{m=1,2 \ldots} \frac{\lambda_{\text {eff }}^{2}}{m !} \Gamma\left(\hbar \omega-E_{0}+m \hbar \omega_{0}\right)\right]
\end{aligned}
$$

In this formula $n(\omega)$ is the refractive index for rr-P3HT that has been extracted from [34]. Already UV/Vis measurements could show that a nonnegligible amount of nonaggregated domains or defects are present in the spin-coated P3HT films. For this reason, fits were also performed using the index of refraction of regiorandom P3HT (rra-P3HT). Results are summarized in the Supplemental Material [26]. They show the same trends but slightly reduced absolute values. Consequently, values presented here can be seen as upper limits due to the ideal rr-P3HT approximation in the refractive index. $\omega$ is the optical frequency, $m$ is the vibrational number, $\lambda_{\text {eff }}^{2}$ is the effective Huang-Rhys parameter, $E_{0}$ is the $0-0$ transition position, $\hbar \omega_{0}=0.175 \mathrm{eV}$ is the energy of the oscillator to which the electronic transition couples, $\alpha$ is a constant that respects disorder, and $\Gamma$ is a Gaussian function. $\alpha$ is a quantitative measure for disorder in the H-aggregate model, since the term is decoupled in the progression formula.

Before discussing the PL results in more detail, we should point out again that the analysis of the ET system is impossible due to the discussed absorption overlap. On the contrary, the observed essential decrease of this transition can be supposed to be interpreted as efficient energy transfer that reduces the PL at this vibronic transition. Generally, it can be observed that P3HT shows almost identical results both on a glass substrate and on mesoporous $\mathrm{TiO}_{2}$ (see Table III). Values for $\alpha$ are identical within the standard deviation for all samples except the ED system. For this system, $\alpha$ is about $20 \%$ smaller compared to $\mathrm{P} 3 \mathrm{HT}$ on $\mathrm{TiO}_{2}$. This reduction means less energetic disorder, or rather a higher spatial correlation, of site energies [14], which correlates with the smallest value for the Gaussian distribution of site energies $\sigma$. Additionally, the vibronic transitions of the other systems are slightly blueshifted compared to the PCBA system. From this finding we infer that PCBA enhances intermolecular interaction in the polymer, while increasing crystallinity as well. The polymer responsible for emission is found to be a species from the aggregate, which is different from absorption, including contributions of nonaggregated polymers [12] as well. Most interestingly $\lambda_{\text {eff }}^{2}$ is closer to the single chain limit $\left(\lambda_{\text {eff }}^{2}=1\right)$ for both the tBP- and the PCBA-based systems than for other systems. This means that the exciton coherence length increases along the polymer chain while the coherent transport across different chains is enhanced. This is in good agreement with the higher intermolecular bandwidth. The effective Huang-Rhys factor is a measure for intrachain interaction, which is not explicitly considered in the pure H-aggregate case. Since there is always an interplay between inter- and intramolecular interaction, the effective Huang-Rhys factor would be more precisely determined within the framework of the more general HJ-aggregate theory. However the value obtained in the Franck-Condon progression model can be seen as an upper limit, and the relative tendencies are described correctly.

TABLE III. Fitting parameters from a Franck-Condon progression fit using Eq. (3) including the Huang-Rhys factor $\lambda_{\text {eff }}^{2}$, the disorder parameter $\alpha$, and the width $\sigma$ of the Gaussians. Mesoporous $\mathrm{TiO}_{2}$ on glass is used as surface matrix. Corresponding plots can be found in the

\begin{tabular}{|c|c|c|c|c|c|}
\hline Sample & $\alpha$ & $\lambda_{\text {eff }}^{2}$ & $\sigma$ & $0-0$ transition $(\mathrm{eV})$ & $0-1$ transition $(\mathrm{eV})$ \\
\hline P3HT (glass only) & $0.41 \pm 0.01$ & $1.38 \pm 0.02$ & $0.048 \pm 0.0004$ & 1.85 & 1.69 \\
\hline $\mathrm{P} 3 \mathrm{HT}$ & $0.41 \pm 0.01$ & $1.38 \pm 0.02$ & $0.052 \pm 0.0005$ & 1.86 & 1.69 \\
\hline tBP-P3HT & $0.40 \pm 0.01$ & $1.24 \pm 0.02$ & $0.047 \pm 0.0004$ & 1.86 & 1.68 \\
\hline D131-tBP/LiTFSI-P3HT & $0.48 \pm 0.12$ & $1.39 \pm 0.02$ & $0.056 \pm 0.0034$ & 1.86 & 1.68 \\
\hline PCBA-tBP-P3HT & $0.32 \pm 0.01$ & $1.24 \pm 0.02$ & $0.046 \pm 0.0004$ & 1.84 & 1.67 \\
\hline SQ2-tBP-P3HT & $0.23 \pm 0.01$ & $1.39 \pm 0.02$ & $0.048 \pm 0.0004$ & 1.86 & 1.68 \\
\hline
\end{tabular}
Supplemental Material [26]. 


\section{Photoinduced absorption spectroscopy}

To get further insights into the charge generation across the hybrid interface in the ED, ET, and DO systems, quasisteady state PIA measurements at $T=10 \mathrm{~K}$ are employed. These low-temperature measurements allow for a much better signalto-noise ratio, and the polaron yield is generally enhanced (see Supplemental Material [26]). The PIA characteristics of P3HT are also well known in literature $[13,47]$ when deposited onto $\mathrm{TiO}_{2}$, allowing for in-depth analysis of results obtained with our model systems [48]. The first result of our PIA study that has to be mentioned is that polaron characteristics of neat P3HT films on glass substrates are below the detection limit of our setup, owing to the conclusion that charge separation within pure $\mathrm{P} 3 \mathrm{HT}$ is only marginal. Only if there is a suitable donor/acceptor energy offset for exciton dissociation do the polaron signatures of P3HT arise [Fig. 6(a)]. Photoabsorption (PA) is very broad from 1.2 to $1.9 \mathrm{eV}$, with a clear peak signature at $1.25 \mathrm{eV}$. This peak is assigned as the localized intrachain polaron state $[13,47]$, while another delocalized interchain state has been previously described to occur around $1.8 \mathrm{eV}$. This energy is not fixed since it depends on the interchain interaction, which potentially results in a shift of its energetic position. One reason for the spectral broadness can be a contribution of triplet state absorption around $1.45 \mathrm{eV}$ [13]. This effect is enhanced and peaks broaden if there is energetic disorder in the material. As discussed in the previous section, such energetic disorder is present in all model systems under investigation in this study. In Fig. 6(a) the polaron signature for $\mathrm{P} 3 \mathrm{HT}$ on $\mathrm{TiO}_{2}$ is clearly enhanced when exciting with a 405-nm instead of 532-nm laser, where the aggregated polymer absorbs. The relative enhancement in polaron yield is even higher than suggested by the figure since the 405-nm measurement was performed at seven times lower light intensity than the $532 \mathrm{~nm}$ measurements $\left(\sim 6 \mathrm{~mW} \mathrm{~cm}^{-2}\right.$ compared to $\sim 40 \mathrm{~mW} \mathrm{~cm}^{-2}$ ).

We start our analysis with a comparison of P3HT on bare and dye-sensitized $\mathrm{TiO}_{2}$. Our first observation is that the ED system is the only system which delivers higher polaron yields when the aggregated polymer is excited (see Supplemental Material [26]), though the behavior and the signal amplitude are comparable to the pure $\mathrm{TiO}_{2}-\mathrm{P} 3 \mathrm{HT}$ interface (i.e., little absorption by delocalized compared to localized polarons). A different situation is observed for the DO system, for which the localized polaron signature becomes indistinct under a much more relevant delocalized polaron absorption, with a maximum around $1.6 \mathrm{eV}$. This value is close to the triplet signature, which has been previously described for rra-P3HT [13]. These findings are in accordance with results from PL measurements, which have indicated that the DO system contains the most structurally and energetically disordered polymer signatures. The ET system shows not only a much stronger photobleach (PB) effect, but also the highest polaron yield. Additionally, the increase from low- to high-energy PA is clear, suggesting contributions from more delocalized polarons. This, however, cannot be resolved in a well-defined peak since the PB signal of SQ2 overlaps with the polymer absorption at $1.79 \mathrm{eV}$. Nevertheless, the absorption range of the polymer and the dye in the ET system is still complementary, so it is possible to perform PIA measurements for dye-only
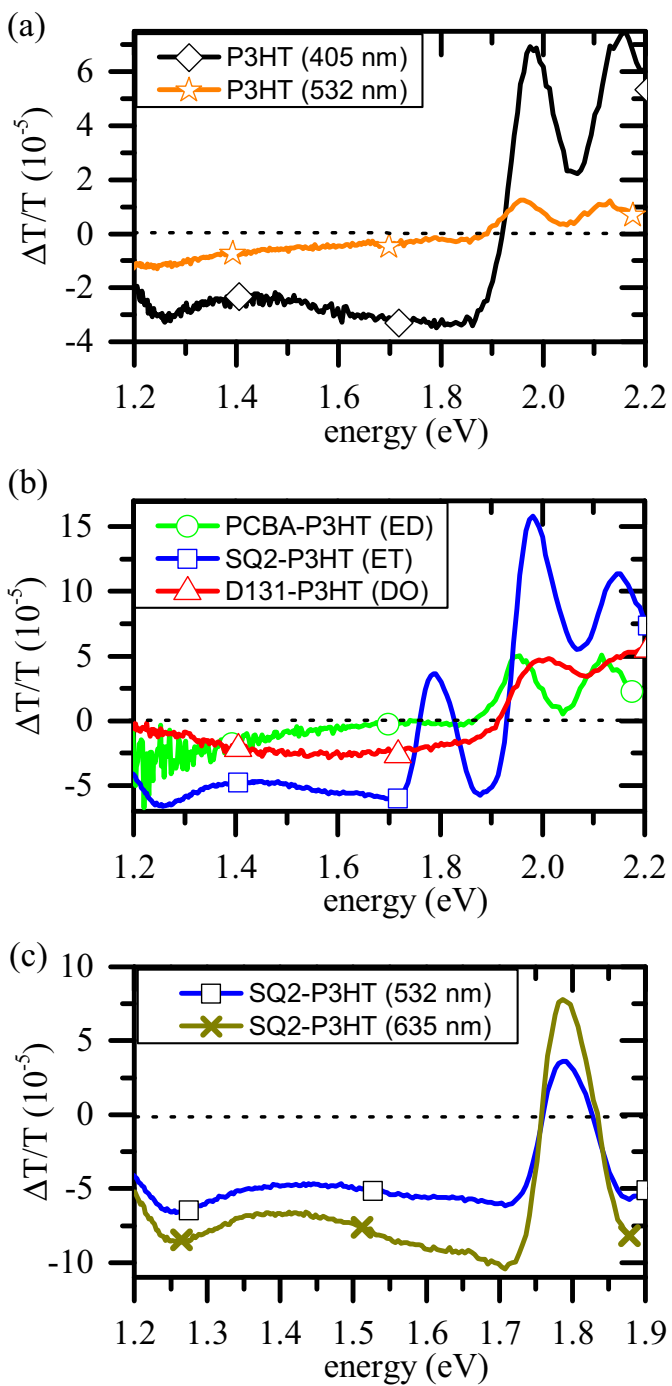

FIG. 6. (Color online) Photoinduced absorption measured at $T=10 \mathrm{~K}$ for (a) P3HT on $\mathrm{TiO}_{2}$ and different pump wavelengths (405 nm/532 nm), (b) dye-modified $\mathrm{TiO}_{2}$ and an excitation at $532 \mathrm{~nm}$, and (c) SQ2 dye-modified $\mathrm{TiO}_{2}$ and different pump wavelength $(532 \mathrm{~nm} / 635 \mathrm{~nm})$. The main contribution of the signal can be found in the in-phase signal of the lock-in amplifier, such that we can simplify the discussion to the R-channel.

excitation using a 635-nm laser [Fig. 6(c)]. At this wavelength, there is virtually no P3HT absorption, so excitons are only generated in the dye. Surprisingly, the signal is enhanced compared to the 532-nm case, although the incident laser intensity $\left(\sim 30 \mathrm{~mW} / \mathrm{cm}^{2}\right)$ is approximately $25 \%$ lower. This behavior reflects the high efficiency for electron injection from SQ2 into $\mathrm{TiO}_{2}$, on the one hand, and the efficient hole transfer to P3HT on the other hand. In other words, these wavelength-dependent results suggest that resonant energy transfer from P3HT to SQ2 with subsequent separation of the SQ2 exciton works at a lower efficiency than charge generation upon direct SQ2 excitation.

When analyzing the PB signals among the different systems, we observe that the PB features are quantitatively different, but the behavior and the dimensions are comparable for 
all systems. The 0-0 and 0-1 transition of P3HT is blueshifted by roughly $10 \mathrm{meV}$ when $\mathrm{TiO}_{2}$ is present, compared to the flat glass substrate. At $T=10 \mathrm{~K}$ the spectrum shows a redshift by 40-50 meV compared to room temperature measurements (see Supplemental Material [26]), and the redshift is $10-40 \mathrm{meV}$ when exciting at $532 \mathrm{~nm}$ compared to $405 \mathrm{~nm}$. A possible explanation for such a behavior is a Stark effect due to the influence of an electric field on the oscillator strength of the absorbing molecules. However, if we compare these nonconsistent shifts among the different systems to UV/Vis results, it is very likely that different polymer crystal structures explain this behavior by different lattice distances such that the nonresonant dispersion interaction can be different. This argument is also used to explain such shifts between low- and room temperature measurements [11]. In contrast, there are also indications that a Stark effect is present, which can be seen in the PB of the dye molecules, most dominantly for $\mathrm{SQ} 2$. Since electron injection into $\mathrm{TiO}_{2}$ upon dye excitation is many orders of magnitude faster [49] than the microsecond time resolution of our quasi-cw-PIA setup, we expect that there is virtually no excited dye (i.e., the PB is not attributed to the presence of excitons in some of the dye molecules [50]). However, it is possible that the dye can be positively charged if not all holes are transferred to the polymer within $1 \mu \mathrm{s}$, resulting in a $\mathrm{PB}$ of the dye. We additionally observe that the $\mathrm{PB}$ is positioned at $1.79 \mathrm{eV}$, which is redshifted $70 \mathrm{meV}$ compared to the absorption peak of SQ2, which is centered at $1.86 \mathrm{eV}$. This could also be attributed to a first-order Stark effect, which arises if there is a change in the molecular dipole moment $\vec{\mu}$ when the dye is in an excited state compared to the ground state. The energy level shift $\Delta E$ is then given by:

$$
\Delta E=-\vec{\mu} \cdot \vec{F}
$$

where $\vec{F}$ is the electric field. Although there is a kind of orientational disorder given by the mesoporous surface, dye molecules are sandwiched between a positively charged polymer and a negatively charged metal oxide, so that the local electric field is pointing toward the metal oxide. Furthermore, the orientation of the dye molecule with respect to the local interface is determined by the $\mathrm{COOH}$ group that links it to the $\mathrm{TiO}_{2}$, so that the dot product of the molecular dipole moment and the local electric field is expected to be very similar at all spots on the hybrid interface. Temperature effects on the $\mathrm{PB}$, introduced by too high illumination intensities, can be ruled out, as discussed in the Supplemental Material [26]. Note, however, that further experiments, like electroabsorption spectroscopy measurements, are necessary to clarify the role of a potential Stark effect at the interface. These experiments are expected to yield fascinating results in the future but are not in the scope of this paper.

\section{DISCUSSION}

In $\mathrm{Sec}$. III C we could show that mesoporous $\mathrm{TiO}_{2}$ only has marginal effect on the polymer crystallinity compared to plane glass substrates. This is not in accordance with previous observations by Kandada et al. [15]. The authors report a noticeable amount of disorder introduced by the mesoporous surface. In our point of view, this observation largely depends on the amount of pore filling, molecular weight, processing conditions, and $\mathrm{TiO}_{2}$ composition. Different preparation conditions, as well as the amount of pore filling, are especially decisive for the amount of nonaggregated polymer domains. In this paper, we were not interested in these factors, in that we have not optimized our systems for this purpose. In fact, it was important to keep processing factors comparable for the different model systems.

Our measurements suggest that $\mathrm{TiO}_{2}$ induces polymer disorder mainly at the first few nanometers away from the hybrid interface. This is the reason why surface modification has a massive impact on the molecular arrangement in hybrid solar cells. Within this study we show that a surface modification can enhance the exciton bandwidth by almost $50 \%$, while the absorption spectra are blueshifted. In other words, the intermolecular $\pi-\pi$ interaction is enhanced while the amount of disorder is increased. To interpret this behavior correctly, it has to be considered that the exciton bandwidth is a relative measure. In a polymer crystal there is always an interplay between inter- and intramolecular interaction. The more relevant the former, the less strong is the latter if the order/disorder ratio stays approximately constant. Otherwise one has to take this fact into account. For this reason, we infer from our results that surface modifiers can reduce the conjugation length along the polymer backbone by the introduction of disorder. This behavior can be explained by locally well-ordered domains that are separated from each other due to the presence of such surface modifiers, either directly because of an imperfect coverage (see Fig. 7) or indirectly due to the introduction of dipoles or surface roughness.

The effective Huang-Rhys factor, extracted from PL spectra, also decreases slightly on a $\mathrm{TiO}_{2}$ surface that has been decorated with interfacial modifiers. Considering a given uncertainty of the fits, the neglect of electron scatterings and lattice imperfections and the fact that one should use the more general $\mathrm{HJ}$-aggregate model for the quantitative determination of $\lambda_{\text {eff }}^{2}$, a Franck-Condon analysis still seems

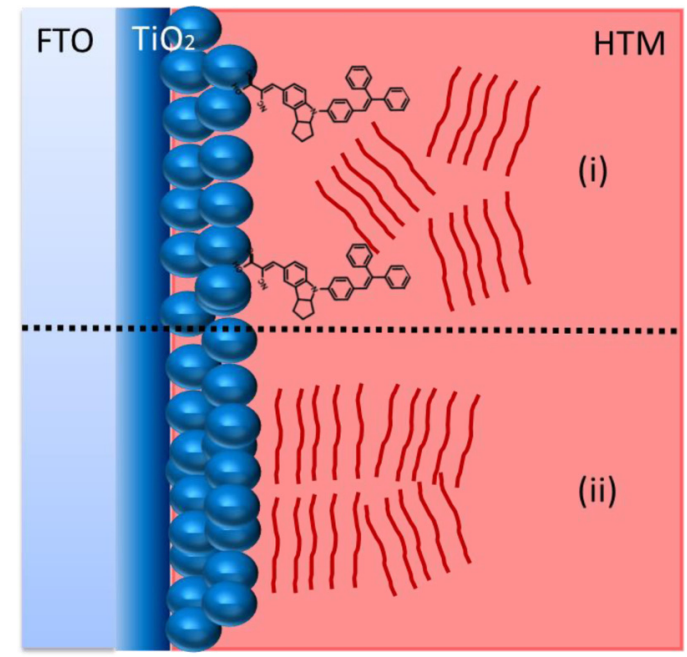

FIG. 7. (Color online) (i) Scheme for how small surface molecule modifications on a titanium surface can influence the polymer arrangement in hybrid solar cells compared to a nonmodified case (ii) 
to be justified for relative comparisons. Additionally, it has to be mentioned that absorption and emission commonly occur at different sites in the polymer, with emission going through crystalline species only. This results in a systematic overestimation of $\lambda_{\text {eff }}^{2}$ so that the given values can be seen as an upper limit only [11]. Our result of a more disordered polymer structure with enhanced intermolecular interaction is in good accordance with observations by Lin et al. [51], who describe an islandlike polymer formation on titanium surfaces in presence of tBP. This study enables us to generalize and extend these observations to other molecules and bring it into agreement with a more sophisticated theoretical model.

The second fundamental question that is addressed within this paper is the electronic communication between dye molecule and polymer at a hybrid interface. For the development of a future high-efficiency hybrid solar cell, it is necessary to gain a fundamental understanding of how charge generation upon photon absorption in the polymer can be maximized. For this purpose, two fundamentally different mechanisms can be employed at the hybrid interface. Excitons in the polymer can be split at the polymer-dye interface, as represented in the ED system, or the excitation energy can be transferred from the polymer to the dye via a FRET, upon which charge separation works the same as for direct excitation of the dye. This is the underlying mechanism for the polymer photocurrent contribution in our ET system. To understand major limitations of both, we have included a dye/polymer combination which fully suppresses photocurrent contributions of the polymer due to a high LUMO of the dye (i.e., the polymer acts as HTM only). The highest power conversion efficiency of all model systems is seen for the ET system due to the highest photocurrent contributions out of the polymer. We attribute this to the high efficiency of charge separation from excited dyes. If the excitation energy is transferred from the polymer to the dye, this efficient mechanism can be taken advantage of, which we see as the reason for the much higher polymer photocurrent contribution in the ET system compared to the ED system. This is in accordance with PIA results, suggesting that hole transfer from the dye to the polymer is much more efficient than electron transfer from the polymer to the dye. However, $V_{\mathrm{OC}}$ and $\mathrm{FF}$ are highest for the system where the polymer is a hole transporter only. For this system, we observe the highest charge collection efficiency, or rather the weakest electric field dependency of charge separation. Taking into account that this system has shown the highest degree of polymer disorder among the three model systems, we conclude that the polymer morphology is not the reason for the low performance of current state-of-the-art hybrid solar cells. This is further supported by the fact that the ED systems exhibit the strongest field dependency of charge carrier extraction, although it exhibits the highest crystalline polymer. Nevertheless, after all other factors at the hybrid interface are optimized, we are convinced that polymer crystallinity remains an important factor for improving mobilities, in particular locally at the hybrid interface. In particular, it is expected that the polymer order strongly affects charge extraction from the interface and thus can play a role in reducing charge recombination. Furthermore fine-tuning of the polymer crystallinity enables one to control the FRET overlap integral for maximizing its efficiency in the charge generation process.

Recently it has been shown by Falke et al. [52] that charge transfer is much more enhanced if it is in an adiabatic limit i.e., the coupling between electronic and nuclear degrees of freedom is coherent, such that the probability for charge transfer is large if the LUMO of donor and acceptor are brought into resonance. In that case, charges can be more delocalized within such noncovalently bound compounds. This effect is not supposed to occur in hybrid systems since vibronic modes are at higher energies for inorganic metal oxides. This idea is supported by similar observations by Kandada et al. [53], who claim that ultrafast ET between donor and acceptor is the primary process, while nonadiabatic charge transfer is a secondary and less efficient mechanism. The resulting lack of delocalization away from the interface is even enhanced in metal oxides due to localized surface states, which lead to low-energy charge transfer states, as demonstrated in the theoretical approach by $\mathrm{Wu}$ et al. [54]. This picture is complicated by the combined theoretical and experimental work of Forrest et al. [55,56], who suggest that a hybrid charge transfer state is present as a coulombically bound charge pair at the organic/inorganic interface. However, taking our results into account, recombination due to charge localization can be reduced if the exciton is separated on a dye molecule, from which charge injection into the metal oxide works more efficiently. Additionally, such a dye molecule acts as a physical spacer between electrons in the metal oxide and holes on the polymer, so that its Coulomb interaction is reduced and charges can travel more efficiently to the electrodes.

\section{CONCLUSION}

According to our results, ET between polymer and dye is by far more efficient in terms of photocurrent generation than exciton separation between polymer and dye, which is supported by findings in the literature for another system [9]. This is partly attributed to a strong field dependency of the charge separation efficiency, as is apparent from charge collection probability measurements. Our results further suggest that this field dependency can only be completely switched off when exciton separation between polymer and dye is suppressed. Furthermore, this seems to be a prerequisite for achieving high open-circuit voltages. Our results are consistent with the findings published by Moon et al. [57] that outstandingly high photocurrents can be achieved in an ET system, which, however, exhibit a comparably low open-circuit voltage.

By using the $\mathrm{H}$-aggregate model, we can confirm the picture of Lin et al. [51] of an island-shaped polymer structure when adding tBP. Based on that, we infer that the conjugation length along the polymer backbone is reduced, while the relative interchain interaction is enhanced, suggesting the presence of small polymer crystallites separated by regions of highly disordered polymer chains. Within our model study, the effect of the polymer disorder is eclipsed by the much stronger impact of the charge separation mechanism on device performance. However, local polymer disorder plays a crucial role for the exact nature of the energetic landscape, both for exciton migration toward the interface, potentially including a FRET, and for charge transport away from the interface. 
Energy transfer between the polymer and the dye appears to be the most promising strategy to activate the photocurrent contribution of the polymer. Therefore, the absorption spectrum of the dye has to be matched with the emission spectrum of the polymer to allow for maximization of the Förster overlap integral. To realize this and suppress exciton separation between dye and polymer at the same time, the dye should exhibit suitable highest occupied molecular orbital (HOMO) and LUMO locations that make electron injection from the polymer into the dye less favorable than ET. This appears to be a necessary condition to allow high open-circuit voltages. Furthermore, the molecular structure of the dye should be optimized to induce crystallization of the polymer close to the interface. Thus, hole polarons can be rapidly removed from the interface after being injected from the dye into the polymer. We are convinced that a combined analysis of charge separation and the recombination mechanism on the one side and the local polymer disorder on the other hand will be necessary to further push the efficiencies of hybrid solar cells.

\section{ACKNOWLEDGMENTS}

This paper was funded through the German Research Foundation (DFG) Project "Limitation" SCHE 634/10-1 in the project "Identification and overcoming loss mechanisms in nanostructured hybrid solar cells-pathways toward more efficient devices" and in the "SPP1355: Elementary processes of organic photovoltaics." P.E. was supported by the German Academic Exchange Service (DAAD) through a travel grant. J.W. acknowledges funding by the Carl Zeiss Foundation.
[1] H. Hintz, H. J. Egelhaaf, L. Lüer, J. Hauch, H. Peisert, and T. Chassé, Chem. Mater. 23, 145 (2010).

[2] H. Sun, J. Weickert, H. C. Hesse, and L. Schmidt-Mende, Solar Energy Materials and Solar Cells 95, 3450 (2011).

[3] H. J. Snaith and L. Schmidt-Mende, Adv. Mater. 19, 3187 (2007).

[4] G. Grancini, R. S. Santosh Kumar, A. Abrusci, H.-L. Yip, C.-Z. Li, A.-K. Y. Jen, G. Lanzani, and H. J. Snaith, Adv. Funct. Mater. 22, 2160 (2012).

[5] J. Weickert and L. Schmidt-Mende, APL Mater. 1, 020901 (2013).

[6] I. K. Ding, N. Tétreault, J. Brillet, B. E. Hardin, E. H. Smith, S. J. Rosenthal, F. Sauvage, M. Grätzel, and M. D. McGehee, Adv. Funct. Mater. 19, 2431 (2009).

[7] A. Abrusci, I. K. Ding, M. Al-Hashimi, T. Segal-Peretz, M. D. McGehee, M. Heeney, G. L. Frey, and H. J. Snaith, Energy Environ. Sci. 4, 3051 (2011).

[8] W. Zhang, R. Zhu, F. Li, Q. Wang, and B. Liu, J. Phys. Chem. C 115, 7038 (2011).

[9] N. Humphry-Baker, K. Driscoll, A. Rao, T. Torres, H. J. Snaith, and R. H. Friend, Nano Lett. 12, 634 (2011).

[10] J. Weickert and L. Schmidt-Mende, in Organic Photovoltaics: Materials, Device Physics, and Manufacturing Technologies, edited by Christoph Brabec, Ullrich Scherf, and Vladimir Dyakonov (Wiley-VCH Verlag GmbH \& Co. KGaA, Weinheim, Germany, 2014), Chap. 15, pp. 465-494.

[11] J. Clark, J.-F. Chang, F. C. Spano, R. H. Friend, and C. Silva, Appl. Phys. Lett. 94, 163306 (2009).

[12] J. Clark, C. Silva, R. H. Friend, and F. C. Spano, Phys. Rev. Lett. 98, 206406 (2007).

[13] R. Österbacka, C. P. An, X. M. Jiang, and Z. V. Vardeny, Science 287, 839 (2000).

[14] F. Paquin, H. Yamagata, N. J. Hestand, M. Sakowicz, N. Bérubé, M. Côté, L. X. Reynolds, S. A. Haque, N. Stingelin, F. C. Spano, and C. Silva, Phys. Rev. B 88, 155202 (2013).

[15] A. R. S. Kandada, S. Guarnera, F. Tassone, G. Lanzani, and A. Petrozza, Adv. Funct. Mater. 24, 3094 (2014).

[16] R. Noriega, J. Rivnay, K. Vandewal, F. P. V. Koch, N. Stingelin, P. Smith, M. F. Toney, and A. Salleo, Nat. Mater. 12, 1038 (2013).,

[17] W. H. Howie, F. Claeyssens, H. Miura, and L. M. Peter, J. Am. Chem. Soc. 130, 1367 (2008).
[18] Y. Vaynzof, D. Kabra, L. Zhao, P. K. H. Ho, A. T.-S. Wee, and R. H. Friend, Appl. Phys. Lett. 97, 033309 (2010).

[19] J. Weickert, F. Auras, T. Bein, and L. Schmidt-Mende, J. Phys. Chem. C 115, 15081 (2011).

[20] J. S. Huang, T. Goh, X. K. Li, M. Y. Sfeir, E. A. Bielinski, S. Tomasulo, M. L. Lee, N. Hazari, and A. D. Taylor, Nat. Photonics 7, 479 (2013).

[21] G. K. Mor, S. Kim, M. Paulose, O. K. Varghese, K. Shankar, J. Basham, and C. A. Grimes, Nano Lett. 9, 4250 (2009).

[22] C. Goh, S. R. Scully, and M. D. McGehee, J. Appl. Phys. 101, 114503 (2007).

[23] J. Weickert, E. Zimmermann, J. B. Reindl, T. Pfadler, J. A. Dorman, A. Petrozza, and L. Schmidt-Mende, APL Mater. 1, 042109 (2013).

[24] A. Abrusci, R. S. Santosh Kumar, M. Al Hashimi, M. Heeney, A. Petrozza, and H. J. Snaith, Adv. Funct. Mater. 21, 2571 (2011).

[25] H. Kallel, G. Latini, F. Paquin, R. Rinfret, N. Stingelin, and C. Silva, arXiv:1007.3035 [cond-mat.mtrl-sci].

[26] See Supplemental Material at http://link.aps.org/supplemental/ 10.1103/PhysRevB.91.035304 for delivering an overview over solar cell statistics, a more detailed description of the PL analysis and additional PIA measurements, which solidify the description in the main manuscript.

[27] E. Zimmermann, P. Ehrenreich, T. Pfadler, J. A. Dorman, J. Weickert, and L. Schmidt-Mende, Nat. Photon 8, 669 (2014).

[28] R. S. S. Kumar, G. Grancini, A. Petrozza, A. Abrusci, H. J. Snaith, and G. Lanzani, Opt. Express 21, A469 (2013).

[29] B. Qi and J. Wang, Phys. Chem. Chem. Phys. 15, 8972 (2013).

[30] S. R. Cowan, A. Roy, and A. J. Heeger, Phys. Rev. B 82, 245207 (2010).

[31] R. A. Street, M. Schoendorf, A. Roy, and J. H. Lee, Phys. Rev. B 81, 205307 (2010).

[32] L. J. A. Koster, V. D. Mihailetchi, R. Ramaker, and P. W. M. Blom, Appl. Phys. Lett. 86, 123509 (2005).

[33] O. J. Korovyanko, R. Österbacka, X. M. Jiang, Z. V. Vardeny, and R. A. J. Janssen, Phys. Rev. B 64, 235122 (2001).

[34] P. J. Brown, D. S. Thomas, A. Köhler, J. S. Wilson, J.-S. Kim, C. M. Ramsdale, H. Sirringhaus, and R. H. Friend, Phys. Rev. B 67, 064203 (2003). 
[35] F. Paquin, G. Latini, M. Sakowicz, P.-L. Karsenti, L. Wang, D. Beljonne, N. Stingelin, and C. Silva, Phys. Rev. Lett. 106, 197401 (2011).

[36] S. D. Oosterhout, M. M. Wienk, S. S. van Bavel, R. Thiedmann, L. Jan Anton Koster, J. Gilot, J. Loos, V. Schmidt, and R. A. J. Janssen, Nat. Mater 8, 818 (2009).

[37] H. Sirringhaus, P. J. Brown, R. H. Friend, M. M. Nielsen, K. Bechgaard, B. M. W. Langeveld-Voss, A. J. H. Spiering, R. A. J. Janssen, E. W. Meijer, P. Herwig, and D. M. de Leeuw, Nature 401, 685 (1999).

[38] F. C. Spano and C. Silva, Annu. Rev. Phys. Chem. 65, 477 (2014).

[39] C. Scharsich, R. H. Lohwasser, M. Sommer, U. Asawapirom, U. Scherf, M. Thelakkat, D. Neher, and A. Köhler, J. Polym. Sci., Part B: Polym. Phys. 50, 442 (2012).

[40] F. C. Spano, J. Clark, C. Silva, and R. H. Friend, J. Chem. Phys. 130, 074904 (2009).

[41] R. J. Kline, M. D. McGehee, E. N. Kadnikova, J. Liu, J. M. J. Fréchet, and M. F. Toney, Macromolecules 38, 3312 (2005).

[42] S. Zhang, X. Yang, C. Qin, Y. Numata, and L. Han, J. Mater. Chem. A 2, 5167 (2014).

[43] F. C. Spano, Acc. Chem. Res. 43, 429 (2009).

[44] F. C. Spano, J. Chem. Phys. 122, 234701 (2005).

[45] H. Yamagata and F. C. Spano, J. Chem. Phys. 136, 184901 (2012).

[46] G. Louarn, M. Trznadel, J. P. Buisson, J. Laska, A. Pron, M. Lapkowski, and S. Lefrant, J. Chem. Phys. 100, 12532 (1996).
[47] X. M. Jiang, R. Österbacka, O. Korovyanko, C. P. An, B. Horovitz, R. A. J. Janssen, and Z. V. Vardeny, Adv. Funct. Mater. 12, 587 (2002).

[48] K. M. Noone, N. C. Anderson, N. E. Horwitz, A. M. Munro, A. P. Kulkarni, and D. S. Ginger, ACS Nano 3, 1345 (2009).

[49] J. Teuscher, J.-D. Décoppet, A. Punzi, S. M. Zakeeruddin, J.-E. Moser, and M. Grätzel, J. Phys. Chem. Lett. 3, 3786 (2012).

[50] P. V. Kamat, S. Das, K. George Thomas, and M. V. George, Chem. Phys. Lett. 178, 75 (1991).

[51] J.-F. Lin, G.-Y. Tu, C.-C. Ho, C.-Y. Chang, W.-C. Yen, S.-H. Hsu, Y.-F. Chen, and W.-F. Su, ACS Applied Materials \& Interfaces 5, 1009 (2013).

[52] S. M. Falke, C. A. Rozzi, D. Brida, M. Maiuri, M. Amato, E. Sommer, A. De Sio, A. Rubio, G. Cerullo, E. Molinari, and C. Lienau, Science 344, 1001 (2014).

[53] A. R. S. Kandada, G. Grancini, A. Petrozza, S. Perissinotto, D. Fazzi, S. S. K. Raavi, and G. Lanzani, Sci. Rep. 3, 02073 (2013).

[54] G. Wu, Z. Li, X. Zhang, and G. Lu, App. Mat. 5, 2649 (2014).

[55] A. Panda, C. K. Renshaw, A. Oskooi, K. Lee, and S. R. Forrest, Phys. Rev. B 90, 045303 (2014).

[56] C. K. Renshaw and S. R. Forrest, Phys. Rev. B 90, 045302 (2014).

[57] S.-J. Moon, E. Baranoff, S. M. Zakeeruddin, C.-Y. Yeh, E. W.-G. Diau, M. Gratzel, and K. Sivula, Chem. Commun. 47, 8244 (2011). 